\title{
Cell-based and cell-free biocatalysis for the production of D-glucaric acid
}

\author{
Lu-Zhou Chen', Si-Ling Huang ${ }^{2}$, Jin Hou ${ }^{3}$, Xue-Ping Guo ${ }^{2}$, Feng-Shan Wang ${ }^{1,4}$ and Ju-Zheng Sheng ${ }^{1,4^{*}}$ (D)
}

\begin{abstract}
D-Glucaric acid (GA) is a value-added chemical produced from biomass, and has potential applications as a versatile platform chemical, food additive, metal sequestering agent, and therapeutic agent. Marketed GA is currently produced chemically, but increasing demand is driving the search for eco-friendlier and more efficient production approaches. Cell-based production of GA represents an alternative strategy for GA production. A series of synthetic pathways for GA have been ported into Escherichia coli, Saccharomyces cerevisiae and Pichia pastoris, respectively, and these engineered cells show the ability to synthesize GA de novo. Optimization of the GA metabolic pathways in host cells has leapt forward, and the titer and yield have increased rapidly. Meanwhile, cell-free multi-enzyme catalysis, in which the desired pathway is constructed in vitro from enzymes and cofactors involved in GA biosynthesis, has also realized efficient GA bioconversion. This review presents an overview of studies of the development of cell-based GA production, followed by a brief discussion of potential applications of biosensors that respond to GA in these biosynthesis routes.
\end{abstract}

Keywords: D-Glucaric acid, Escherichia coli, Yeast, Metabolic engineering, Cell-free synthetic biology, Biosensor

\section{Background}

D-Glucaric acid (GA), also called glucarate or saccharic acid, is a naturally occurring aldaric acid in animals and several types of fruits and vegetables $[1,2]$. This compound has been used in many fields, including the chemical, food, pharmaceutical, and therapeutic industries [3]. From 2004, GA was classified as a "top value-added chemical from biomass" by the United States Department of Energy because of its potential applications as a material for making biodegradable detergents and biodegradable polymers such as nylons and plastics [4]. As it combines well with metal ions, it is also used as an imaging agent in tumor observation, a surfactant in sewage treatment, and a decolorizer in the treatment of synthetic dyes [5-7]. In medicine, GA is used to reduce cholesterol

\footnotetext{
*Correspondence: Shengjuzheng@sdu.edu.cn

${ }^{1}$ Key Laboratory of Chemical Biology of Natural Products (Ministry

of Education), School of Pharmaceutical Sciences, Cheeloo College

of Medicine, Shandong University, Jinan 250012, China

Full list of author information is available at the end of the article
}

and suppress tumor development [1]. GA could also enhance human immunity and reduce cancer risks if used as a food additive [8].

GA is a by-product of the glucuronic acid metabolism pathway (Fig. 1) in animal and plant cells [9]. This quantitatively minor route of glucose metabolism catalyzes the conversion of glucose to glucuronic acid (GlcA), ascorbic acid, and pentoses, and also provides biosynthetic precursors (nucleotide sugars) to synthesize glycans. In this pathway, glucose-6-phosphate (G6P) formed from glucose is isomerized into glucose-1-phosphate (G1P) by phosphoglucomutase. G1P reacts with uridine triphosphate, catalyzed by uridine $5^{\prime}$-diphosphate-glucose (UDP-Glc) pyrophosphorylase, to form UDP-Glc, which is oxidized in a two-step process by an $\mathrm{NAD}^{+}$-dependent UDP-Glc dehydrogenase to UDP-GlcA. UDP-GlcA is hydrolyzed to form UDP and D-GlcA. Then the latter undergoes a three-step conversion reaction through the intermediates D-glucuronic- $\gamma$-lactone and D-glucaro- $\gamma$ lactone to form GA. However, GA is found in plant and

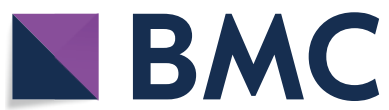

c The Author(s) 2020. This article is licensed under a Creative Commons Attribution 4.0 International License, which permits use, sharing, adaptation, distribution and reproduction in any medium or format, as long as you give appropriate credit to the original author(s) and the source, provide a link to the Creative Commons licence, and indicate if changes were made. The images or other third party material in this article are included in the article's Creative Commons licence, unless indicated otherwise in a credit line to the material. If material is not included in the article's Creative Commons licence and your intended use is not permitted by statutory regulation or exceeds the permitted use, you will need to obtain permission directly from the copyright holder. To view a copy of this licence, visit http://creativeco mmons.org/licenses/by/4.0/. The Creative Commons Public Domain Dedication waiver (http://creativecommons.org/publicdomain/ zero/1.0/) applies to the data made available in this article, unless otherwise stated in a credit line to the data. 


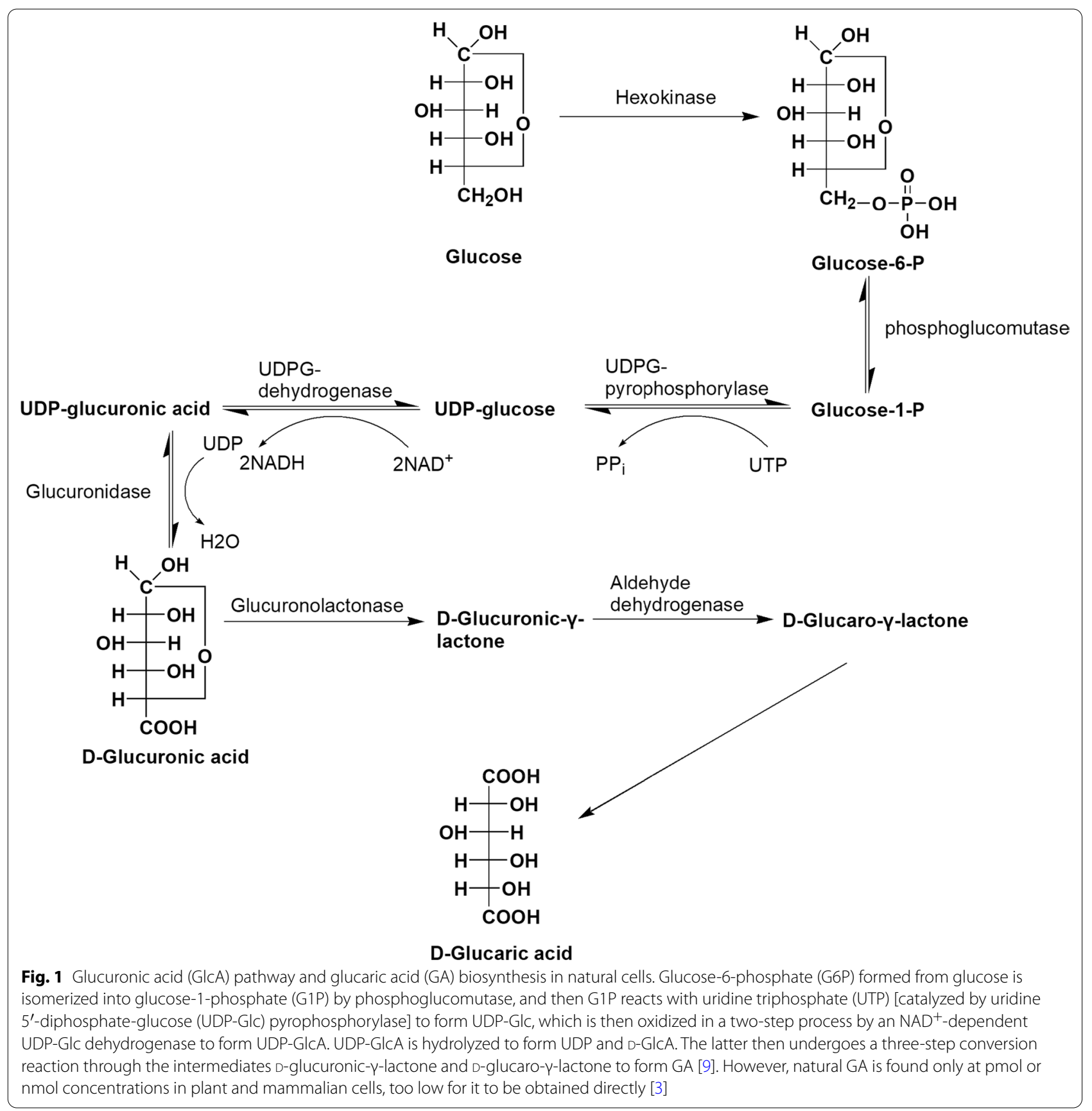

mammalian cells at only pmol or nmol concentrations, which is too low for it to be obtained directly [3].

Thus, marketed GA is currently produced chemically. First, a nonselective oxidation reaction using nitric acid was developed [10]. Though optimized, the molar ratio of nitric acid to glucose was 4:1, causing great damage to the environment and had a low conversion from glucose to GA ( $<50 \%$ yield) [11]. This situation was not changed until the 1960s when the compound 2,2,6,6-tetramethyl-1-piperidinyloxy (TEMPO) was discovered [12]. This material functions as a catalyst in the oxidation of alcohols to aldehydes. It means the reaction can be performed in milder conditions, with production of more of the target compound (70-90\% yield) [13-16]. However, because the chemical oxidation method for GA production from glucose is not selective, it is difficult to obtain a single compound, and the production of nitrogen compound byproducts is environmentally unfriendly 
[11]. Therefore, it is highly desirable to develop environmentally-friendly and cost-effective biosynthesis strategies to produce GA. Metabolic engineering and in vitro multiple-enzyme catalysis are effective strategies, and have leapt forward in recent years [17]. Indeed, metabolic engineering and in vitro multiple-enzyme catalysis are effective strategies, and have leapt forward in recent years [17]. This review provides an introduction to synthetic-biology-based methodologies for high-yield GA-biosynthesizing cells (Escherichia coli and yeast) (Table 1), followed by a discussion of in vitro multipleenzyme catalysis approaches to produce GA.

\section{Construction and optimization of heterologous GA synthesis pathways in $E$. coli}

\section{Construction of heterologous GA synthesis pathways in $E$.} coli

The first successful example of GA biosynthesis in microbes using a metabolic engineering approach was the manipulation of recombinant $E$. coli to produce GA (Fig. 2a). This Gram-negative bacterium is a very versatile host for the production of heterologous proteins, such as those within the natural GA synthesis pathway. In 2009, Moon et al. successfully constructed a recombinant $E$. coli strain producing GA from glucose by heterologously co-expressing three enzymes: myo-inositol-1-phosphate synthase (Ino1) from Saccharomyces cerevisiae, which converts G6P into myo-inositol-1-phosphate (I1P); myoinositol oxygenase (MIOX) from mouse, which converts myo-inositol (MI) into GlcA with the consumption of one molecule of oxygen; and Pseudomonas syringae uronate dehydrogenase (Udh), which converts the GlcA into GA using $\mathrm{NAD}^{+}$as a cofactor. The final titer of GA from glucose using this strain reached $1.13 \mathrm{~g} / \mathrm{L}$ and the yield was $0.113 \mathrm{~g} / \mathrm{g}$ glucose [18]. However, it was found that MIOX is intrinsically unstable, which directly limited the process of MI conversion to GlcA [18]. Furthermore, a considerable fraction of the carbon source (such as glucose in M9 medium) is used for cell growth and only a small fraction enters the artificially constructed GA production pathway [18], which demonstrates that the competition of various metabolic pathways in cells is also one of the factors that affects GA production.

Meanwhile, some researchers have tried to use other sugars as substrates for synthesis of GA. Sucrose was used as the substrate rather than D-glucose [19]. Sucrose has the advantages of being low cost and easy to obtain [20]. Compared with the pathway starting from D-glucose, the pathway starting from sucrose requires three additional enzymes, invertase (CscA), sucrose permease $(\mathrm{CscB})$ and $\mathrm{D}$-fructokinase (CscK) from E. coli strain W (Fig. 2b). These enzymes could make E. coli have the ability of consuming sucrose and convert sucrose into D-glucose and D-fructose; the former monosaccharide is then used for GA synthesis, while the D-fructose acts as a carbon source that improves the cell growth compared with use of $\mathrm{D}$-glucose as the sole carbon source.

\section{Synthetic scaffolds for multiple enzymes in E. coli}

Co-expression of multiple enzymes on a scaffold in one cell is an effective strategy to increase GA synthesis efficiency. Use of synthetic scaffolds in GA-synthesizing $E$.

Table 1 Summary of bio-based production of D-glucaric acid. Modified from Ref. [69]

\begin{tabular}{|c|c|c|c|c|c|c|c|}
\hline & Year & Hosts & Genes & Carbon sources & Titer $(\mathrm{g} / \mathrm{L})$ & Yield (g/g) & Refs. \\
\hline \multirow[t]{13}{*}{ In vivo } & 2009 & E. coli BL21 Star (DE3) & ino1, MIOX, udh & Glucose & 1.13 & 0.113 & [18] \\
\hline & 2010 & E. coli BL21 Star(DE3) & ino1, MIOX, udh & Glucose & 2.50 & 0.250 & {$[22]$} \\
\hline & 2014 & E. coli MG1655 & ino 1, SUMO-MIOX, udh & Myo-inositol & 4.85 & 0.449 & {$[26]$} \\
\hline & 2015 & E. coli IB1486 & ino1, SUMO-MIOX, udh & Glucose & 1.56 & 0.124 & [44] \\
\hline & 2017 & E. coli L19S & ino 1, MIOX, udh & Glucose & $>0.8$ & - & {$[45]$} \\
\hline & 2018 & E. coli K-12 & ino1, MIOX, udh & Glucose & 1.98 & 0.198 & {$[48]$} \\
\hline & 2018 & E. coli BL21 Star(DE3) & $\csc B, \csc A, \csc$, ino 1, MIOX, udh, suhB & Sucrose & 1.42 & 0.27 & {$[19]$} \\
\hline & 2020 & E. coli BL21 Star(DE3) & ino1, MIOX, udh, suhB & Glucose & 5.35 & 0.467 & {$[40]$} \\
\hline & 2016 & S. cerevisiae CEN.PK2-1D opi1 $\triangle$ & ino1, inm, MIOX, udh & Glucose & 0.98 & 0.33 & {$[50]$} \\
\hline & 2016 & S. cerevisiae CEN.PK2-1D opi1 & inol, inm, MIOX, udh & Glucose, myo-inositol & 1.6 & - & {$[50]$} \\
\hline & 2016 & P. pastoris GS115 & MIOX, udh & Glucose, myo-inositol & 6.61 & - & {$[50]$} \\
\hline & 2018 & S. cerevisiae BY4741 opi1 & ino 1, MIOX, udh & Glucose, myo-inositol & 6.0 & - & {$[55]$} \\
\hline & 2020 & S. cerevisiae & ino1, inm, MIOX, udh, vhB & Glucose & 6.38 & 0.128 & {$[58]$} \\
\hline \multirow[t]{4}{*}{ In vitro } & 2016 & Soluble enzymes & xyn, $A G$, udh & Hemicellulose & - & - & {$[67]$} \\
\hline & 2019 & Soluble enzymes & SP, PGM1, MIPS, IMPASE, MIOX, udh, nox & Sucrose & 7.31 & 0.75 & {$[64]$} \\
\hline & 2020 & Soluble enzymes & AxyAgu115A, GOOX & Xylan & - & - & {$[68]$} \\
\hline & 2020 & Immobilized enzymes & PGM1, IPS, IMP, MIOX, udh, nox & Glucose-1-phosphate & $1.70 \pm 0.04$ & 0.202 & [69] \\
\hline
\end{tabular}




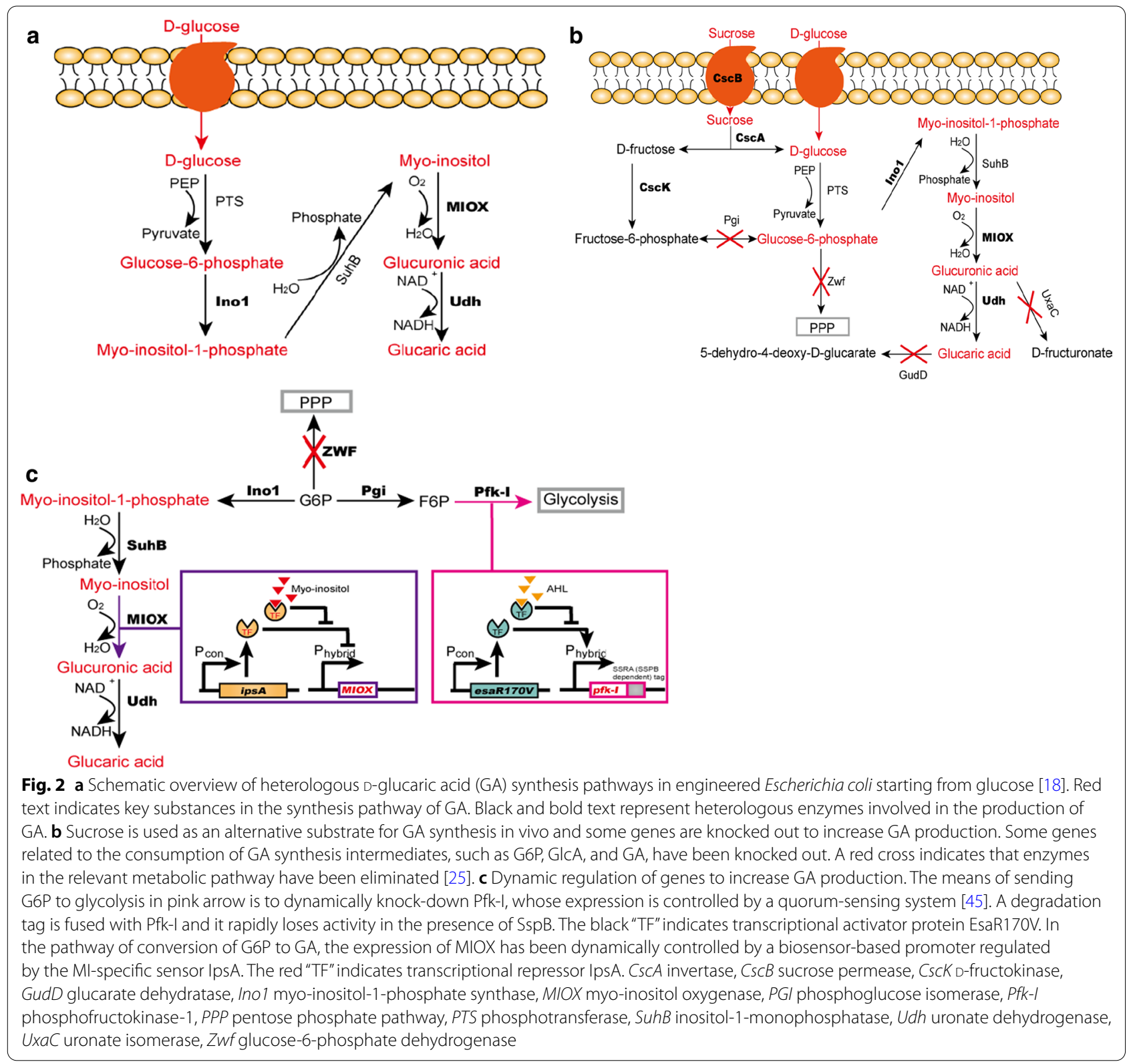

coli strains allows greater channeling of intermediates through improved flux, stabilization of intermediates, and protection from other reactions [21]. A scaffold carrying specific ligands combining the functional domains of Ino1 and MIOX was constructed in vivo, which achieved enzyme colocalization and greatly improved the activity of MIOX. The production of GA starting from glucose reached $2.5 \mathrm{~g} / \mathrm{L}, 5$ times higher than when the enzymes without the scaffold were expressed from the same vectors and the yield of GA was $0.25 \mathrm{~g} / \mathrm{g}$ glucose [22].

\section{Optimization of metabolic routes through elimination of competing enzymes}

The knockout of competing genes has been used to improve the conversion ratio of strains for some chemical products [23]. G6P is a primary material for cell growth, and takes part in the Enter-Dudoroff and Embden-Meyerhoff-Parnas pathways in the cell [24]. After introducing the GA production pathway into $E$. coli, it was found that a large part of G6P entered the glycolytic pathways and was used for cell growth at the initial stage (Fig. 2b). Considering it is an intermediate in the heterologous synthesis of GA, genes encoding glucose-6-phosphate 
dehydrogenase $(z w f)$ and phosphoglucose isomerase ( $p g i)$ were knocked out from the E. coli chromosome, resulting in more G6P accumulation in the cell and either glycerol, xylose, or arabinose was used as carbon sources instead of glucose for cell growth [25]. Furthermore, genes encoding uronate isomerase $(u x a C)$ and glucarate dehydratase $(\operatorname{gudD})$ were knocked out to increase the amount of the intermediate D-GlcA and decrease the consumption of the product GA [26].

\section{Optimization of the rate-limiting enzyme-MIOX}

It has been become generally accepted that MIOX is the rate-limiting enzyme within the artificial GA biosynthesis pathway in E. coli. Natural MIOX is part of a pathway for the catabolism of inositol in kidneys [2]. It was first identified in 1957 from extracts of rat kidney [27]. However, its catalytic mechanism was not determined until the 1980 s because of its poor stability in vitro: this nonheme di-iron enzyme employs a unique four-electron transfer at its $\mathrm{Fe}(\mathrm{II}) / \mathrm{Fe}(\mathrm{III})$ coordination sites, and the reaction proceeds through the attack of the iron center by diatomic oxygen followed by abstraction of the MI hydrogen atom [28]. Therefore, the reaction catalyzed by MIOX needs $\mathrm{Fe}^{2+}$ and cysteine both in vitro (for purified recombinant MIOX) and in recombinant E. coli [29]. Strategies to make this artificial GA biosynthesis pathway more effective include improving the expression level of soluble MIOX in recombinant cells and using mutants with better catalytic activity instead of wild-type MIOX. In E. coli, mouse MIOX, a protein from eukaryotic cells, possibly fails to fold properly, instead forming aggregates and precipitating as inclusion bodies, resulting in a limited level of soluble MIOX. A small ubiquitin-related modifier (SUMO)-tag, a commonly used fusion tag to enhance expression and solubility of recombinant proteins in E. coli [30], was fused at the N-terminus of MIOX and the yield of GA increased $125 \%$ compared with the use of wild-type MIOX [26].

Directed evolution has emerged in the past few years as a powerful tool that is widely used to modify the properties of enzymes [31-34]. Usually, in the absence of sufficient information about the protein structure and catalytic mechanism, common mutation methods such as error-prone PCR and chemical mutagens are applied to obtain a large number of mutants of the desired protein [35]. The key to the success of directed evolution is a suitable high-throughput screening method depending on selective protein properties that can be used to screen the desired protein efficiently [36]. There are two methods to screen MIOX. One is to use the native metabolism of $E$. coli to couple the activity of MIOX with the growth rate of the strain [26]. E. coli cannot grow when MI is used as the sole carbon source. However, when MIOX converts
MI into GlcA, E. coli can consume GlcA as the carbon source and grow. Therefore, the screening of high-activity MIOX mutants can be achieved by screening the cellular growth rate when using MI as the sole carbon source. Although the authors did not identify a high-activity MIOX mutant using this screening method, they unexpectedly discovered a DNA fragment that could increase MI transport [26]. In this better GA producing host, the wild-type MIOX gene was mutated and a 941-bp DNA insertion fragment occurred downstream of the MIOX terminator. GA production was obviously decreased when the 941-bp DNA fragment was removed. The authors analyzed the DNA fragment and found that the proteins encoded by this DNA fragment could enhance the transportation of MI into the cell [26]. Another method of screening for desired MIOX mutants is using a GA-responsive biosensor [37]. Based on synthetic tools such as biosensors, high-throughput screening strategies using individual recombinant $E$. coli cells as a sorting section are playing growing roles in the directed evolution of enzymes involved in GA biosynthesis. A biosensor that responds to GA has been designed and successfully used in identification of desired MIOX orthologs and GA-producing strains $[37,38]$. Recently, several highly active mutants of MIOX have been identified through an effective high-throughput screening approach (a one-pot two-strain system) based on a GA biosensor system [37]. D82Y and S173N mutants of MIOX displayed approximately 3.8- and 2.7-times higher than the wild-type activity toward $\mathrm{MI}$ in a broad $\mathrm{pH}$ range (pH 3-6).

Ribosome binding site (RBS) optimization is another strategy to enhance GA production by coordinating the expression of enzymes [39]. The activity ratio of Ino1 to MIOX was improved from 1:0.40 to 1:3.41, which greatly enhanced the GA titer starting from glucose, to $4.56 \mathrm{~g} / \mathrm{L}$, compared with the unoptimized strain $(3.42 \mathrm{~g} / \mathrm{L})$ and the yield of GA was $0.467 \mathrm{~g} / \mathrm{g}$ glucose [40].

\section{Dynamic control of GA synthetic pathways}

Dynamic control is an important tool in metabolic engineering that allows global optimization of flux of carbon in vivo. Phosphofructokinase (Pfk-I) is a critical enzyme controlling the flux of G6P into glycolysis that influences cell growth. Meanwhile, G6P is an important intermediate in GA synthetic pathways, and in vivo self-regulation of Pfk-I expression, G6P could be switched between glycolysis and GA metabolic pathway (Fig. 2c). Additionally, it would maximize GA yields and minimize the need for manual intervention in the fermentation processes. Firstly, 10Sa RNA (ssrA) was added to the coding sequence of $p f k-I$ to enable dynamic degradation of Pfk-I in the cell [41]. This degradation tag would result in the slow degradation of Pfk-I in the absence of stringent 
starvation protein $B(\mathrm{Ssp} B)$ and rapid degradation in the presence of SspB which tethers target proteins to the protease ClpXP, resulting in the accumulation of G6P, the precursor of MI, in the cells [42, 43]. Based on this, a relationship between the activity of Pfk-I and the expression of SspB was established: the more SspB was expressed, the less Pfk-I was present in the cell. The expression of SspB was controlled by an inducible promoter. In this way, the strain of overexpressing $\mathrm{SspB}$ redirected more G6P to the GA intermediate MI by controlling the degradation of Pfk-I. As expected, E. coli carrying this gene showed the $1.56 \mathrm{~g} / \mathrm{L}$ of the GA titer from glucose, a $42 \%$ improvement over the strain without $\operatorname{ssp} B$ and the yield of GA was $0.124 \mathrm{~g} / \mathrm{g}$ glucose [44].

In a development of this approach, a modified and selfconstructed quorum sensing (QS) system was developed to achieve dynamic expression of Pfk-I protein [45]. QS is a common mechanism by which bacteria regulate gene expression based on cell density using signal molecules [46]. The synthesis of capsular polysaccharide (CPS) is a typical process controlled by QS, and the histone acetyltransferase Esa1 that acts as a transcriptional activator of CPS synthesis is induced by the level of 3-oxohexanoylhomoserine lactone (AHL) [47]. As mentioned before, Pfk-I takes part in many important reactions in the process of cell growth, and knocking out Pfk-I causes the accumulation of G6P. Thus, by controlling the expression of Pfk-I, "cell growth" and "GA production" modes could be achieved (Fig. 2c). Initially, the cells would be in "cell growth" mode when Pfk-I was expressed in the cells under the control of the promoter of esaS $\left(\mathrm{P}_{\text {esas }}\right)$ in the presence of regulation by the protein activator EsaR170V. Then, when the cell density reached a desired value, expression of Pfk-I would stop as the AHL interacted with the EsaR170V, resulting in low transcription

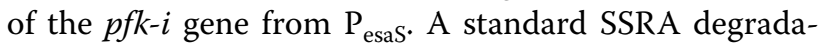
tion tag was appended to the C-terminus of Pfk-I altering the half-life of which to ensure the Pfk-I protein could be removed rapidly when the transcription halted. Using the QS system to control the expression of Pfk-I, the "cell growth" mode would be switched to the "GA production" mode when the cell population reached a desired level. In this engineered system, the final MI titer increased 5.5fold and the GA titer fourfold higher compared with the strain with $p f k-i$ controlled by its native promoter [45].

Lastly, the dynamic control method was also used to control the expression of MIOX, to avoid loss of catalytic activity over time in E. coli (Fig. 2c). Briefly, a hybrid promoter that could sense the concentration of MI to control the expression of MIOX was used [48]. In the absence of MI, the transcription factor IpsA could bind with this promoter and recruit RNA polymerase to activate $M I O X$ gene expression [49]. In the presence of MI, MI binds to
IpsA, causing the IpsA to dissociate from DNA, hence decreasing $M I O X$ gene expression. Then, based on this system, a hybrid promoter that could sense MI with the opposite mechanism was engineered: expression of the gene of interest was repressed by blocking access to RNA polymerase in the absence of MI. Binding of MI to IpsA would cause structural changes of IpsA which would dissociate from the promoter, activating expression of the gene of interest. Using the MI-responsive promoter to control MIOX expression, the GA titer from glucose was increased 2.5-fold compared with the unregulated MIOX control. Finally, when combining the dynamic control method to regulate the expression of Pfk-I and MIOX, the final GA titer was $1.98 \mathrm{~g} / \mathrm{L}$, nearly fivefold increase compared with that when only Pfk-I was regulated $(0.4 \mathrm{~g} / \mathrm{L})$ and the yield of GA was $0.198 \mathrm{~g} / \mathrm{g}$ glucose [48].

\section{Construction and optimization of heterologous GA synthesis pathways in yeast}

Engineering E. coli cells for GA production requires mitigation of the limitations that arise from the inherent toxicity of GA toward E. coli [30]. Yeast cells are more resistant to toxicity resulting from a low $\mathrm{pH}$ environment than E. coli. Thus, the GA biosynthesis pathway has been introduced into yeasts such as $S$. cerevisiae and Pichia pastoris [50]. The former species has "generally recognized as safe" status, and is widely used in the metabolic engineering field for production of industrial materials such as bioethanol, and bulk and fine chemicals $[51,52]$. P. pastoris is increasing in use as an alternative host to $S$. cerevisiae because it can be fermented to very high density, enabling high level production of target compounds [53]. Unlike E. coli, yeasts have native forms of Ino1 and inositol monophosphatase (Inm1) that convert G6P to MI (Fig. 3). Therefore, introducing the heterologous enzymes MIOX and Udh could give cells GA producing ability. However, conversion from MI to GlcA catalyzed by MIOX is less active than the other enzyme-catalyzed steps in the artificial GA biosynthesis pathway in recombinant yeast [50]. To explore candidate genes encoding MIOX that were better suited for heterologous overexpression in yeast, $M I O X$ genes from Arabidopsis thaliana were employed to replace the role of MIOX from Mus musculus, and introduced into the GA biosynthesis pathway [50]. Compared with MIOX from Mus musculus, higher GA production, $\sim 0.5 \mathrm{~g} / \mathrm{L}$, was observed when the recombinant $S$. cerevisiae carried the $A$. thaliana miox gene. Chen et al, tried to solve the problem of low catalytic efficiency of MIOX by integrating the MIOX gene from $A$. thaliana and $u d h$ from $P$. syringae into a delta $(\delta)$-sequence. The $\delta$-sequence is usually employed to increase the expression of protein under the control of strong promoters, such as the 


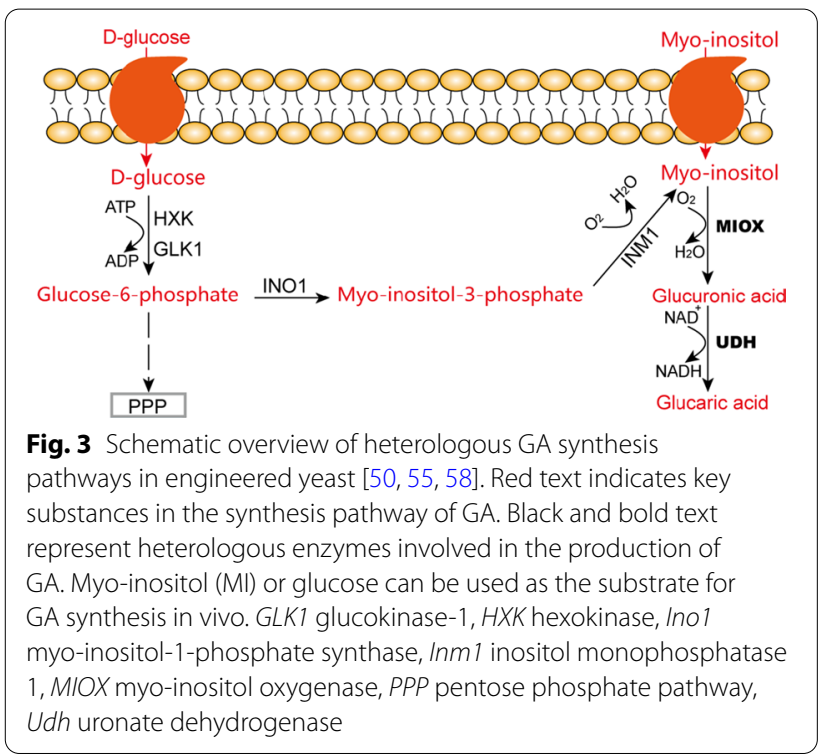

promoter of glyceraldehyde-3-phosphate dehydrogenase [54]. The engineered strain produced $3.8 \mathrm{~g} / \mathrm{L}$ GA from myo-inositol in shake flask culture, 7.04 times higher than that of the strain without integration of genes into the $\delta$-sequence. The final GA starting from glucose and myo-inositol reached $6 \mathrm{~g} / \mathrm{L}$ after the fed-batch fermentation [55]. In 2020, to identify better candidates for GA production in S. cerevisiae, proteins in the UniProt database with sequence similarity to MIOX family members together with Udh were, respectively, introduced into recombinant yeast cells harboring the pathway form glucose to GA [56]. In this study, 25 novel homologues were characterized, and displayed the ability to convert MI to GA together with Udh. It is worth mentioning that the recombinant cells expressing Talaromyces marneffei MIOX produced more GA, $1.76 \pm 0.33 \mathrm{~g} / \mathrm{L}$ GA from $20 \mathrm{~g}$ glucose $/ \mathrm{L}$ and $10 \mathrm{~g} / \mathrm{L} \mathrm{MI}$, than cells expressing the oftenused Arabidopsis thaliana variant AtMiox4.

In a recent report, it was found that titers of GA could be improved by introducing Vitreoscilla hemoglobin into cells to increase the amount of dissolved oxygen [57]. In the conversion of MI to GlcA, MIOX acts as a monooxygenase and oxygen is needed in the reaction process. The increase of cellular oxygen might increase oxygen availability for this reaction and/or accelerate cell metabolism and/or growth and thus cause more efficient GA production [58]. It is worth mentioning that when P. pastoris was employed as the host, recombinant strains produced GA from glucose and MI up to $6.61 \pm 0.30 \mathrm{~g} / \mathrm{L}$, which is also better than the titer achieved in recombinant $E$. coli [40].

The production of GA correlated with MI availability in recombinant yeast [50]. The final production increased when the medium was supplemented with MI, which indicated that insufficient MI was being synthesized, and this created a bottleneck in GA synthesis in yeast. Unlike in E. coli, in yeasts, MI is an important material (produced by endogenous Inol and Inm). In this process, the expression of Ino1 is controlled by the negative regulation of OPI1. The expression of Ino1 was greatly increased when the gene opi1 was knocked out, resulting in a substantial increase in production of the GA precursor MI [59].

\section{Cell-free multi-enzyme catalysis approaches to produce $\mathbf{G A}$}

In vitro multi-enzyme catalysis is another candidate route for biosynthesis of GA. Cell-free multi-enzyme catalysis usually involves construction of a single pathway with several enzymes and coenzymes in vitro. Cell-free multienzyme catalysis approaches for GA production are commonly considered to be efficient and environmentally-friendly, and to be a practical solution to the problems posed by fermentation [60]. These issues, such as low conversion rate and unbalanced pathway fluxes, have hampered industrial-scale production of GA using metabolically engineered $E$. coli or yeast strains. Meanwhile, cell-free multi-enzyme catalysis systems have advantages such as high reaction efficiency, easy control of reaction conditions and product separation, and a small environmental footprint [61-63].

In 2019, an in vitro GA biosynthesis method using sucrose as substrate and seven enzymes was established (Fig. 4a) [64]. Briefly, sucrose phosphorylase (SP) converted sucrose into G1P and D-fructose. Then, G1P was transformed into G6P by phosphoglucomutase (PGM). Next, Ino1 converted G6P into I1P. The I1P was dephosphorylated into MI, and finally oxidized to GA by MIOX and Udh. NADH oxidase (Nox) could achieve the recycling of NADH during the GA synthesis process. By optimizing the reaction conditions and enzyme composition, the GA yield was up to $0.7 \mathrm{~g} / \mathrm{g}$ sucrose from $17 \mathrm{~g} / \mathrm{L}$ sucrose, and the final concentration of GA was $7.3 \mathrm{~g} / \mathrm{L}$. As Nox could regenerate $\mathrm{NAD}^{+}$, the reaction only needed $2 \mathrm{~g} \mathrm{NAD}^{+} / \mathrm{L}$. This saved costs and enhanced efficiency. Hemicellulose, the second most abundant form of biomass in plant cell walls after cellulose, is an alternative material that can be employed for efficient conversion into GA $[65,66]$. A successful biosynthesis pathway from hemicellulose to GA was constructed (Fig. 4a). The pathway was different from the previously reported pathway constructed in recombinant $E$. coli, and used three enzymes: xylanase (Xyn) from Flavobacterium sp., glcuronidase (AG) from a rumen metagenomic library, and Udh from P. mendocina [67]. These three enzymes were co-localized into a scaffold and the final product 


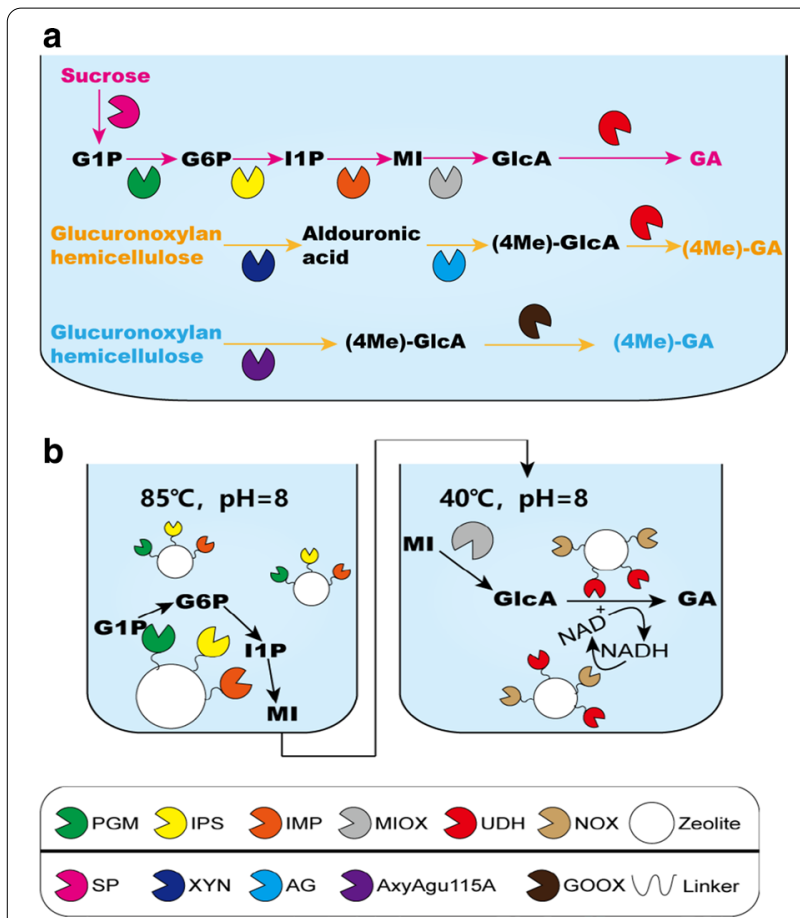

Fig. 4 In vitro cascade for production of GA. Four different methods of synthesizing GA in vitro are represented by different colored text and arrows [64, 67-69]. a Pink text indicates the GA synthesis route starting from sucrose. Sucrose is converted to GIP by SP, and then GA is produced by the action of five enzymes-PGM, IPS, IMP, MIOX, and Udh. Orange text represents the in vitro synthesis route of GA starting from glucurononxylan hemicellulose. Blue text represents a novel synthesis route of GA starting from glucurononxylan hemicellulose. $\mathbf{b}$ Black text represents the in vitro synthesis route of GA starting from G1P. The reaction was divided into two parts: high-temperature and low-temperature systems. The enzymes related to the GA pathway were connected to zeolite via a peptide linker. AG glucuronidase, AxyAgu115A GH115 a-glucuronidase, G1P glucose-1-phosphate, G6P glucose-6-phosphate, GOOX gluco-oligosaccharide oxidase, I1P myo-inositol-1-phosphate, IMP inositol-1-monophosphatase, IPS myo-inositol-3-phosphate, MI myo-inositol, MIOX myo-inositol oxygenase, Nox NADH oxidase, PGM phosphoglucomutase, SP sucrose phosphorylase, $U d h$ uronate dehydrogenase, $X Y N$ xylanase

GA increased by $20 \%$ compared with the case when the three enzymes were free in solution. However, continuous supplementation with the expensive cofactor $\mathrm{NAD}^{+}$ was required in this reaction system, and the soluble xylo-oligosaccharides affect the separation of GA from the reaction system. To avoid these limitations, a novel method of producing GA from hardwood xylan was constructed, in which no supplementation of $\mathrm{NAD}^{+}$was required and the xylan co-product could be easily separated by centrifugation [68]. Briefly, two novel enzymes were introduced into the one-pot reaction (Fig. 4a). A GH115 $\alpha$-glucuronidase (a member of glycoside hydrolase family 115) from Amphibacillus xylanus releases almost all the 4-O-methyl D-GlcA from glucuronoxylan. Then, a Y300A mutant of Sarocladium strictum glucooligosaccharide oxidase converts the glucuronoxylan to $4-O$-methyl D-GlcA. The yield of GA in this process was $0.62 \mathrm{~g} / \mathrm{g}$ 4-O-methyl D-GlcA [68].

Petroll et al. [69] constructed an immobilized multienzyme biosynthesis system, and they successfully overcame the limitations of cell-free catalysis based on immobilization of thermostable enzymes and cofactor regeneration (Fig. 4b). The in vitro GA titer from G1P was $3.0 \pm 0.2 \mathrm{~g} / \mathrm{L}$ for free enzymes, and $1.70 \pm 0.04 \mathrm{~g} / \mathrm{L}$ for immobilized enzymes. Briefly, they divided the GA synthesis reaction into two parts depending on the temperature stability of the enzymes. The thermostable enzymes consisted of phosphoglucomutase (PGM-L), myo-inositol-3-phosphate synthase (IPS-L), and inositol1-monophosphatase (IMP-L), and using these enzymes, G1P was produced from MI at $85{ }^{\circ} \mathrm{C}$. The non-thermostable enzymes, MIOX, Udh, and Nox, converted MI to GA at $40{ }^{\circ} \mathrm{C}$. In addition, a linker peptide was fused at either the $\mathrm{N}$ - or C-terminus of these enzymes so they had good affinity for the silica-based matrix [70].

\section{High-throughput screening approach based on GA biosensor}

It is highly desirable to develop an effective GA concentration screening approach. Promoters responding to $\mathrm{GA}$, and promoters that allow for real-time control of upstream genes, would be an effective solution. Such promoters and their transcriptional regulators would have high value, and enable optimum GA pathway flux and construction of GA biosensors. The biosensors would be powerful during the identification of critical in vivo points for improvement of GA titer, and for directed evolution of enzymes involved in GA biosynthesis. In 2016, Rogers et al. [71] developed a GA biosensor based on the transcription factor CdaR and a 521-bp promoter in E. coli. Two years later, an effective highthroughput screening approach was established, and applied in the identification of highly active mutants of MIOX [37]. An E. coli strain containing the GA biosensor system could act as a GA "detector", and with its help, the GA concentration within medium or a reaction mixture could be ranked. Though this approach is not a quantitative analysis method, it is suitable for high-throughput sorting, and a feasible solution to the lack of a fast and effective GA detection method. Yeasts are candidate host cells that may be better suited for the production of GA than $E$. coli, because of their higher resistance to acid toxicity from GA accumulation in vivo. Unfortunately, GAinducible transcriptional regulators of natural eukaryotic promoters have not been reported, though it is believed that these components would be straightforward and 
powerful tools for yeast metabolic engineering. Transplantation of prokaryotic transcriptional activators into the eukaryotic chassis is a potential solution to this problem [72-74].

\section{Conclusion}

GA is commonly identified as one of the "top valueadded chemicals from biomass", and is currently produced by chemical method. The alternative is bio-based production approaches. Therefore, the development of GA production based on fermentation and metabolically engineered cells or in vitro cell-free biocatalysis has leapt forward in recent years, and GA titers and yield have increased rapidly [18, 19, 26, 40, 51, 64, 69]. Firstly, a series of synthetic pathways for GA have been ported into E. coli, S. cerevisiae, and P. pastoris, and these engineered cells show the ability to synthesize GA de novo. After abundant works to improve the GA metabolic pathways in host cells, GA titers from glucose have reached around $5 \mathrm{~g} / \mathrm{L}$ (from glucose) and $6 \mathrm{~g} / \mathrm{L}$ (from glucose and MI) when E. coli and P. pastoris are used as host cells, respectively.

Probing protein sequence-function relationships of MIOXs then engineering members of this enzyme family would yield MIOX variants with desired enzymatic activity and increased stability, which would be a fundamentally important step in advancing biobased GA production. Furthermore, tools in synthetic biology are changing rapidly, which will be important for improvement of the efficiency of GA biobased production. There has been recent interest in the dynamic regulation of flux through metabolic pathways to overcome some of the issues arising from the introduction of the GA biosynthesis pathway into host cells. In the future, with a range of metabolite sensors responding to GA directly or to intermediate metabolites within the GA biosynthesis pathway being discovered or artificially constructed, control can be implemented at different dynamic timescales.

Briefly, we expect that continuing advances in synthetic biology and protein engineering will allow the design of efficient and environmentally-friendly approaches to produce GA, and meet the requirements of GA application in medicine and industry.

\footnotetext{
Abbreviations

AHL: 3-Oxohexanoyl-homoserine lactone; CPS: Capsular poly-saccharide; CscA: Invertase; CscB: Sucrose permease; CscK: D-Fructokinase; EsaR: Acyl-homoserine-lactone synthase repressor; GA: Glucaric acid; GlcA: Glucuronic acid; GudD: Glucarate dehydratase; G1P: Glucose 1-phosphate; G6P: Glucose-6-phosphate; IMP: Inositol-1-monophosphatas; Inm1: Inositol monophosphatase; Ino1: Myo-inositol-1-phosphate synthase; I1P: Myo-inositol-1-phosphate; MI: Myo-inositol; MIOX: Myo-inositol oxygenase; MIPS: Myo-inositol 1-phosphate synthase; Nox: NADH oxidase; Pgi: Phosphoglucose isomerase; PGM: Phosphoglucomutase; Pfk: Phosphofructokinase; QS: Quorum sensing; SP: Sucrose phosphorylase; RBS: Ribosome binding site; SsrA: 10Sa RNA; SspB:
}

Stringent starvation protein B; SUMO: Small ubiquitin-related modifier; TEMPO: 2,2,6,6-Tetramethyl-1-piperidinyloxy; Udh: Uronate dehydrogenase; UxaC: Uronate isomerase; Zwf: Glucose-6-phosphate dehydrogenase.

\section{Acknowledgements}

We thank Dr. Shuang Zheng (THU) for the helpful discussions.

\section{Authors' contributions}

FW and JS coordinated the manuscript. LC, PG and JS wrote the manuscript. $\mathrm{LC}, \mathrm{SH}$ and $\mathrm{JH}$ designed the figures. All authors read and approved the final manuscript.

\section{Funding}

This work was supported in part by the National Natural Science Foundation of China (Project Nos. 31770845, 31970082 and 31770101), as well as the Fundamental Research Funds for the Central Universities and the Qilu Scholar Program of Shandong University.

\section{Availability of data and materials}

No new data generated in this review.

\section{Ethics approval and consent to participate}

Not applicable.

\section{Consent for publication}

All authors consented on the publication of this work.

\section{Competing interests}

The authors declare that they have no competing interests.

\section{Author details}

${ }^{1}$ Key Laboratory of Chemical Biology of Natural Products (Ministry of Education), School of Pharmaceutical Sciences, Cheeloo College of Medicine, Shandong University, Jinan 250012, China. ${ }^{2}$ Bloomage BioTechnology Corp., Ltd., Jinan 250010, China. ${ }^{3}$ The State Key Laboratory of Microbial Technology, Shandong University, Qingdao 266237, China. ${ }^{4}$ National Glycoengineering Research Center, Shandong University, Jinan 250012, China.

Received: 22 August 2020 Accepted: 2 December 2020

Published online: 10 December 2020

\section{References}

1. Walaszek Z, Szemraj J, Hanausek M, Adams AK, Sherman U. D-Glucaric acid content of various fruits and vegetables and cholesterol-lowering effects of dietary D-glucarate in the rat. Nutr Res. 1996;16:673-81.

2. Moskala R, Reddy CC, Minard RD, Hamilton GA. An oxygen-18 tracer investigation of the mechanism of myo-inositol oxygenase. Biochem Biophys Res Commun. 1981;99:107-13.

3. Polen T, Spelberg M, Bott M. Toward biotechnological production of adipic acid and precursors from biorenewables. J Biotechnol. 2013;167:75-84.

4. Werpy T, Petersen G. Top value added chemicals from biomass volume 1 -results of screening for potential candidates from sugars and synthesis gas. Golden: National Renewable Energy Lab; 2004. https://doi. org/10.2172/15008859.

5. Khaw B, Silva J, Petrov A, Hartner W. Indium 111 antimyosin and Tc-99m glucaric acid for noninvasive identification of oncotic and apoptotic myocardial necrosis. J Nucl Cardiol. 2002;9:471-81.

6. Aury S, Rubini P, Gérardin C, Selve C. Amphiphilic amide derivatives of D-glucaric acid. Synthesis and complexing properties toward lanthanide(III) ions. Eur J Org Chem. 2004;2004:2057-66.

7. Verma P, Shah V, Baldrian P, Gabriel J, Stopka P, Trnka T, et al. Decolorization of synthetic dyes using a copper complex with glucaric acid. Chemosphere. 2004:54:291-5.

8. Marsh CA. Metabolism of D-glucuronolactone in mammalian systems. 2. Conversion of D-glucuronolactone into D-glucaric acid by tissue preparations. Biochem J. 1963;87:82-90. 
9. Kuellmer V. Ascorbic acid. Kirk-Othmer encyclopedia of chemical technology. Atlanta: American Cancer Society; 2001. https://doi. org/10.1002/0471238961.0119031511210512.a01.pub2.

10. Mehltretter $C L$, Rist CE. Sugar oxidation, saccharic and oxalic acids by the nitric acid oxidation of dextrose. J Agric Food Chem. 1953;1:779-83.

11. Smith TN, Hash K, Davey C-L, Mills H, Williams H, Kiely DE. Modifications in the nitric acid oxidation of D-glucose. Carbohydr Res. 2012;350:6-13.

12. Lajzerow J. Structure of 2,2,6,6-tetramethylpiperidin-4-ol-I-oxide nitroxide radical. Acta Crystallogr Sect B Struct Crystallogr Cryst Chem. 1968;24:196-9.

13. Thaburet J-F, Merbouh N, Ibert M, Marsais F, Queguiner G. TEMPOmediated oxidation of maltodextrins and D-glucose: effect of $\mathrm{pH}$ on the selectivity and sequestering ability of the resulting polycarboxylates. Carbohydr Res. 2001;330:21-9.

14. Merbouh N, Francois Thaburet J, Ibert M, Marsais F, Bobbitt JM. Facile nitroxide-mediated oxidations of D-glucose to D-glucaric acid. Carbohydr Res. 2001;336:75-8.

15. Belgsir EM, Schäfer HJ. Selective oxidation of carbohydrates on Nafion ${ }^{\circledR}$ TEMPO-modified graphite felt electrodes. Electrochem Commun. 2001:3:32-5.

16. Ibert M, Fuertès P, Merbouh N, Fiol-Petit C, Feasson C, Marsais F. Improved preparative electrochemical oxidation of D-glucose to D-glucaric acid. Electrochim Acta. 2010;55:3589-94.

17. Radakovits R, Jinkerson RE, Darzins A, Posewitz MC. Genetic engineering of algae for enhanced biofuel production. Eukaryot Cell. 2010;9:486-501.

18. Moon TS, Yoon S-H, Lanza AM, Roy-Mayhew JD, Prather KLJ. Production of glucaric acid from a synthetic pathway in recombinant Escherichia coli. Appl Environ Microbiol. 2009;75:589-95.

19. Qu Y-N, Yan H-J, Guo Q, Li J-L, Ruan Y-C, Yue X-Z, et al. Biosynthesis of D-glucaric acid from sucrose with routed carbon distribution in metabolically engineered Escherichia coli. Metab Eng. 2018;47:393-400.

20. Bruschi M, Boyes SJ, Sugiarto H, Nielsen LK, Vickers CE. A transferable sucrose utilization approach for non-sucrose-utilizing Escherichia coli strains. Biotechnol Adv. 2012;30:1001-10.

21. Dueber JE, Wu GC, Malmirchegini GR, Moon TS, Petzold CJ, Ullal AV, et al. Synthetic protein scaffolds provide modular control over metabolic flux. Nat Biotechnol. 2009;27:753-9.

22. Moon TS, Dueber JE, Shiue E, Prather KLJ. Use of modular, synthetic scaffolds for improved production of glucaric acid in engineered E. coli. Metab Eng. 2010;12:298-305.

23. Tyo KE, Kocharin K, Nielsen J. Toward design-based engineering of industrial microbes. Curr Opin Microbiol. 2010;13:255-62

24. Guo W, Zou L-F, Cai L-L, Chen G-Y. Glucose-6-phosphate dehydrogenase is required for extracellular polysaccharide production, cell motility and the full virulence of Xanthomonas oryzae pv. oryzicola. Microb Pathog. 2015;78:87-94.

25. Shiue E, Brockman IM, Prather KLJ. Improving product yields on D-glucose in Escherichia coli via knockout of pgi and zwf and feeding of supplemental carbon sources. Biotechnol Bioeng. 2015;112:579-87.

26. Shiue E, Prather KLJ. Improving D-glucaric acid production from myoinositol in E. coli by increasing MIOX stability and myo-inositol transport. Metab Eng. 2014;22:22-31.

27. Charalampous FC, Lyras C. Biochemical studies on inositol. IV. Conversion of inositol to glucuronic acid by rat kidney extracts. J Biol Chem. 1957:228:1-13.

28. Reddy CC, Pierzchala P, Hamilton G. Myo-Inositol oxygenase from hog kidney. II. Catalytic properties of the homogeneous enzyme. J Biol Chem. 1981;256:8519-24.

29. Arner RJ, Prabhu KS, Thompson JT, Hildenbrandt GR, Liken AD, Reddy CC. Myo-inositol oxygenase: molecular cloning and expression of a unique enzyme that oxidizes myo-inositol and D-chiro-inositol. Biochem J. 2001:8:313-20.

30. Esposito D, Chatterjee DK. Enhancement of soluble protein expression through the use of fusion tags. Curr Opin Biotechnol. 2006;17:353-8.

31. Collins $\mathrm{CH}$, Arnold FH, Leadbetter JR. Directed evolution of Vibrio fischeri LuxR for increased sensitivity to a broad spectrum of acyl-homoserine lactones: directed evolution of LuxR. Mol Microbiol. 2004;55:712-23.

32. Sen S, Venkata Dasu V, Mandal B. Developments in directed evolution for improving enzyme functions. Appl Biochem Biotechnol. 2007; 143:212-23.
33. Eijsink VGH, Gåseidnes $S$, Borchert TV, van den Burg B. Directed evolution of enzyme stability. Biomol Eng. 2005;22:21-30.

34. Hibbert EG, Dalby PA. Directed evolution strategies for improved enzymatic performance. Microb Cell Fact. 2005;4:29.

35. Marcheschi RJ, Gronenberg LS, Liao JC. Protein engineering for metabolic engineering: current and next-generation tools. Biotechnol J. 2013;8:545-55.

36. You L, Arnold FH. Directed evolution of subtilisin E in Bacillus subtilis to enhance total activity in aqueous dimethylformamide. Protein Eng Des Sel. 1996:9:77-83.

37. Zheng S, Hou J, Zhou Y, Fang H, Wang T-T, Liu F, et al. One-pot two-strain system based on glucaric acid biosensor for rapid screening of myoinositol oxygenase mutations and glucaric acid production in recombinant cells. Metab Eng. 2018;49:212-9.

38. Rogers JK, Guzman CD, Taylor ND, Raman S, Anderson K, Church GM. Synthetic biosensors for precise gene control and real-time monitoring of metabolites. Nucleic Acids Res. 2015;43:7648-60.

39. Liu S, Zhang $X$, Liu F, Xu M, Yang T, Long M, et al. Designing of a cofactor self-sufficient whole-cell biocatalyst system for production of 1,2-amino alcohols from epoxides. ACS Synth Biol. 2019:8:734-43.

40. Su H-H, Peng F, Ou X-Y, Zeng Y-J, Zong M-H, Lou W-Y. Combinatorial synthetic pathway fine-tuning and cofactor regeneration for metabolic engineering of Escherichia coli significantly improve production of D-glucaric acid. New Biotechnol. 2020:59:51-8.

41. Andersen JB, Sternberg C, Poulsen LK, Bjørn SP, Givskov M, Molin S. New unstable variants of green fluorescent protein for studies of transient gene expression in bacteria. Appl Environ Microbiol. 1998;64:2240-6.

42. McGinness KE, Baker TA, Sauer RT. Engineering controllable protein degradation. Mol Cell. 2006;22:701-7.

43. Davis JH, Baker TA, Sauer RT. Small-molecule control of protein degradation using split adaptors. ACS Chem Biol. 2011;6:1205-13.

44. Reizman IMB, Stenger AR, Reisch CR, Gupta A, Connors NC, Prather KLJ. Improvement of glucaric acid production in E. coli via dynamic control of metabolic fluxes. Metab Eng Commun. 2015;2:109-16.

45. Gupta A, Reizman IMB, Reisch CR, Prather KLJ. Dynamic regulation of metabolic flux in engineered bacteria using a pathway-independent quorum-sensing circuit. Nat Biotechnol. 2017;35:273-9.

46. Fuqua WC, Winans SC, Greenberg EP. Quorum sensing in bacteria: the LuxR-Luxl family of cell density-responsive transcriptional regulators. J Bacteriol. 1994;176:7.

47. Minogue TD, Trebra MW, Bernhard F, von Bodman SB. The autoregulatory role of EsaR, a quorum-sensing regulator in Pantoea stewartii ssp. stewartii: evidence for a repressor function. Mol Microbiol. 2002;44:1625-35.

48. Doong SJ, Gupta A, Prather KLJ. Layered dynamic regulation for improving metabolic pathway productivity in Escherichia coli. Proc Natl Acad Sci. 2018:115:2964-9.

49. Baumgart M, Luder K, Grover S, Gätgens C, Besra GS, Frunzke J. IpsA, A novel Lacl-type regulator, is required for inositol-derived lipid formation in Corynebacteria and Mycobacteria. BMC Biol. 2013;11:122.

50. Gupta A, Hicks MA, Manchester SP, Prather KLJ. Porting the synthetic D-glucaric acid pathway from Escherichia coli to Saccharomyces cerevisiae. Biotechnol J. 2016;11:1201-8.

51. Liu Y, Gong X, Wang C, Du G, Chen J, Kang Z. Production of glucaric acid from myo-inositol in engineered Pichia pastoris. Enzyme Microb Technol. 2016;91:8-16.

52. Nevoigt E. Progress in metabolic engineering of Saccharomyces cerevisiae. MMBR. 2008;72:379-412

53. Holmes WJ, Darby RA, Wilks MD, Smith R, Bill RM. Developing a scalable model of recombinant protein yield from Pichia pastoris: the influence of culture conditions, biomass and induction regime. Microb Cell Fact. 2009;8:35.

54. Parekh RN, Shaw MR, Wittrup KD. An integrating vector for tunable, high copy, stable integration into the dispersed Ty $\delta$ sites of saccharomyces cerevisiae. Biotechnol Prog. 1996;12:16-21.

55. Chen N, Wang J, Zhao Y, Deng Y. Metabolic engineering of Saccharomyces cerevisiae for efficient production of glucaric acid at high titer. Microb Cell Fact. 2018;17:67.

56. Marques WL, Anderson LA, Sandoval L, Hicks MA, Prather KLJ. Sequencebased bioprospecting of myo-inositol oxygenase (Miox) reveals new homologues that increase glucaric acid production in Saccharomyces cerevisiae. Enzyme Microb Technol. 2020;140:109623. 
57. Fish PA, Webster DA, Stark BC. Vitreoscilla hemoglobin enhances the first step in 2,4-dinitrotoluene degradation in vitro and at low aeration in vivo. J Mol Catal B Enzym. 2000;9:75-82.

58. Zhang X, Xu C, Liu Y, Wang J, Zhao Y, Deng Y. Enhancement of glucaric acid production in Saccharomyces cerevisiae by expressing Vitreoscilla hemoglobin. Biotechnol Lett. 2020;42:2169-78.

59. Henry SA, Gaspar ML, Jesch SA. The response to inositol: regulation of glycerolipid metabolism and stress response signaling in yeast. Chem Phys Lipid. 2014;180:23-43.

60. Pellis A, Cantone S, Ebert C, Gardossi L. Evolving biocatalysis to meet bioeconomy challenges and opportunities. New Biotechnol. 2018;40:154-69.

61. Petroll K, Kopp D, Care A, Bergquist PL, Sunna A. Tools and strategies for constructing cell-free enzyme pathways. Biotechnol Adv. 2019;37:91-108.

62. Sheldon RA. The limits to biocatalysis: pushing the envelope. Chem Commun. 2018;54:6088-104.

63. Sperl JM, Sieber V. Multienzyme cascade reactions — status and recent advances. ACS Catal. 2018;8:2385-96.

64. Su H, Guo Z, Wu X, Xu P, Li N, Zong M, et al. Efficient bioconversion of sucrose to high-value-added glucaric acid by in vitro metabolic engineering. ChemSusChem. 2019;12:2278-85.

65. Adsul MG, Singhvi MS, Gaikaiwari SA, Gokhale DV. Development of biocatalysts for production of commodity chemicals from lignocellulosic biomass. Bioresour Technol. 2011;102:4304-12.

66. Tuck CO, Perez E, Horvath IT, Sheldon RA, Poliakoff M. Valorization of biomass: deriving more value from waste. Science. 2012;337:695-9.

67. Lee CC, Kibblewhite RE, Paavola CD, Orts WJ, Wagschal K. Production of glucaric acid from hemicellulose substrate by rosettasome enzyme assemblies. Mol Biotechnol. 2016;58:489-96.
68. Vuong TV, Master ER. Enzymatic production of 4-O-methyl D-glucaric acid from hardwood xylan. Biotechnol Biofuels. 2020;13:51.

69. Petroll K, Care A, Bergquist PL, Sunna A. A novel framework for the cellfree enzymatic production of glucaric acid. Metab Eng. 2020;57:162-73.

70. Care A, Petroll K, Gibson ESY, Bergquist PL, Sunna A. Solid-binding peptides for immobilisation of thermostable enzymes to hydrolyse biomass polysaccharides. Biotechnol Biofuels. 2017;10:29.

71. Rogers JK, Church GM. Genetically encoded sensors enable realtime observation of metabolite production. Proc Natl Acad Sci. 2016;113:2388-93.

72. Skjoedt ML, Snoek T, Kildegaard KR, Arsovska D, Eichenberger M, Goedecke TJ, et al. Engineering prokaryotic transcriptional activators as metabolite biosensors in yeast. Nat Chem Biol. 2016;12:951-8.

73. Chen X, Yang X, Shen Y, Hou J, Bao X. Screening phosphorylation site mutations in yeast acetyl-CoA carboxylase using malonyl-CoA sensor to improve malonyl-CoA-derived product. Front Microbiol. 2018;9:47.

74. Ambri F, D'Ambrosio V, Di Blasi R, Maury J, Jacobsen SAB, McCloskey D, et al. High-resolution scanning of optimal biosensor reporter promoters in yeast. ACS Synth Biol. 2020;9:218-26.

\section{Publisher's Note}

Springer Nature remains neutral with regard to jurisdictional claims in published maps and institutional affiliations.
Ready to submit your research? Choose BMC and benefit from:

- fast, convenient online submission

- thorough peer review by experienced researchers in your field

- rapid publication on acceptance

- support for research data, including large and complex data types

- gold Open Access which fosters wider collaboration and increased citations

- maximum visibility for your research: over $100 \mathrm{M}$ website views per year

At BMC, research is always in progress.

Learn more biomedcentral.com/submissions 